\title{
Performance of Early and Late Planting Cotton Genotypes under Agro-Ecological Conditions of Multan, Punjab, Pakistan
}

\author{
Muhammad Iqbal', Muhammad Mahmood Iqbal ${ }^{*}$, Saghir Ahmad ${ }^{1}$, Athar Mahmood ${ }^{2}$, Muhammad \\ Akram ${ }^{1}$, Hammad Husnain ${ }^{1}$, Muhammad Shahid ${ }^{1}$, Saeed Ahmad ${ }^{1}$, Ali Raza ${ }^{1}$, Ansar Hussain ${ }^{3}$, Allah Ditta \\ Abid $^{4}$, Qaisar Abbas ${ }^{5}$, Mussarrat Hussain ${ }^{5}$, Muhammad Akram ${ }^{6}$ and Muhammad Umair Hassan ${ }^{2}$
}

${ }^{1}$ Cotton Research Institute, Multan, Pakistan; ${ }^{2}$ Department of Agronomy, University of Agriculture, Faisalabad, Pakistan; ${ }^{3}$ Ghazi University Dera Ghazi Khan, Pakistan; ${ }^{4}$ Department of Plant Protection, Malair Halt Karachi, Pakistan; ${ }^{5}$ Entomological Research Station, Multan, Pakistan; ${ }^{6}$ Agronomic Research Institute, Faisalabad, Pakistan.

\begin{abstract}
Cotton is an imperious cash crop of Pakistan and change in climatic conditions are continuously limiting its production across the globe. Sowing time is an important agronomic consideration which should be optimized to ensure better cotton yield and quality. Therefore, this study was performed to determine the potential of cotton genotypes under varied planting times at cotton research institute, Multan during 2018 and 2019. The experiment was comprised of four cotton genotypes IUB-13, MNH-1016, MNH-1020 and MNH-1026 and eight different sowing times viz $1^{\text {st }}$ March, $16^{\text {th }}$ March, $1^{\text {st }}$ April, $16^{\text {th }}$ April, $1^{\text {st }}$ May, $16^{\text {th }}$ May, $1^{\text {st }}$ June and $16^{\text {th }}$ June. The experiment was performed in RCBD with split plot arrangement and was repeated thrice. Sowing times were placed in main plot and cultivars were placed in sub plots. Results revealed that seed cotton yield and fiber strength was more when cotton was planted early between $1^{\text {st }}$ March to $16^{\text {th }}$ April compared to late planted cotton ( $1^{\text {st }}$ May to $\left.16^{\text {th }} \mathrm{June}\right)$. Highest seed cotton yield was obtained when cotton was sown on $1^{\text {st }}$ April during first year $\left(3946 \mathrm{~kg} \mathrm{ha}^{-1}\right)$ and $16^{\text {th }}$ March during second year (3307 $\left.\mathrm{kg} \mathrm{ha}^{-1}\right)$. Minimum seed cotton yield $\left(852,299 \mathrm{~kg} \mathrm{ha}^{-1}\right)$ during both years was obtained when cotton was sown on $16^{\text {th }}$ June. Among cotton genotypes highest seed cotton yield was recorded in MNH-1020 during first year (3021 kg ha-1) and MNH-1016 during second year $\left(2150 \mathrm{~kg} \mathrm{ha}^{-1}\right)$. However, minimum seed cotton yield, ginning out turn $(38.9,36.6 \%)$ and fiber length $(27.6,26.8 \mathrm{~cm})$ were recorded in IUB-13 during both years. MNH-1020 had highest ginning out turn (41.1, 39.3\%), fiber strength $(35.88,40.44 \mathrm{~g} /$ tex $)$ and fiber length $(28.7,29.2 \mathrm{~cm})$ during both years. MNH-1020 was also least effected with cotton leaf curl virus when planted on $1^{\text {st }}$ March during first year (25.0\%) and $16^{\text {th }}$ march sowing during second year (3.0\%). In conclusion, cotton cultivar MNH-1020 can sown from $1^{\text {st }}$ March to $16^{\text {th }}$ April in order to get better cotton yield and quality.
\end{abstract}

Received | March 16, 2021; Accepted | June 15, 2021; Published | June 27, 2021

*Correspondence | Muhammad Mahmood Iqbal, Cotton Research Institute, Multan, Pakistan; Email: mahmkhokhar@gmail.com

Citation | Iqbal, M., M.M. Iqbal, S. Ahmad, A. Mahmood, M. Akram, H. Husnain, M. Shahid, S. Ahmad, A. Raza, A. Hussain, A.D. Abid, Q. Abbas, M. Hussain, M. Akram and M.U. Hassan. 2021. Performance of early and late planting cotton genotypes under agro-ecological conditions of Multan, Punjab, Pakistan. Pakistan Journal of Agricultural Research, 34(3): 569-579.

DOI | https://dx.doi.org/10.17582/journal.pjar/2021/34.3.569.579

Keywords | Adaptation, Cotton leaf curl virus, Fiber strength, Germplasm, Ginning out turn

\section{Introduction}

Cotton (Gossypium hirsutum L.) being the king of fibers occupies central position in the modern commerce. It plays an imperative role in Pakistan's economy nonetheless, per hectare yield of Pakistan is lower than the other cotton producing countries. Cotton crop has a share of $0.8 \%$ in national GDP 
while it has a contribution of $4.1 \%$ in total value added in agriculture (GoP, 2020). In the countries like Pakistan which is vulnerable to climate change, selection of climate resilient crop management practices plays an appreciable role to achieve the desired yield (Deho et al., 2012). The wrong selection of management consideration can cause a significant reduction in final yield. Abiotic factors such as rainfall, temperature, and irradiance are the main ecological factors influencing cotton growth and development (Bradow and Davidonis, 2000; Chen et al., 2012). Sowing time is an imperative management option to reduce the impact of abiotic factors like drought and heat stress (Hassan et al., 2020a). Deciding the growing season length through sowing date is of remarkable importance (Huang, 2016; Muhsin et al., 2021) owing to fact it has significant impact on crop vegetative and reproductive growth (Hallikeri et al., 2009). The cotton sown in early season faces the hottest period during their reproduction phase which cause a significant reduction in yield (Rahman et al., 2007). Moreover, in late planted cotton flowering and maturity are exposed to high rainfall, low temperature and shorter growth period which in turn reduce the cotton yield and quality (Elayan et al., 2015).

An ever-increasing global population calls for agricultural production systems and cultivars that are productive in unreliable weather patterns and are more efficient in utilization of resources in scenarios of climate change, heat and drought stress (Hassan et al., 2020b). The cultivation of suitable cultivar plant a crucial role in growth and final productivity (Chattha et al., 2017, 2020; Hassan et al., 2018,2019a, b, 2020c; Ilyas et al., 2021). Varieties with varied yield potentials are available but their production potential could not be attained under field conditions (Reynolds and Tuberosa, 2008). Yield reduction is mainly due to the cultivation of varieties without considering their behavior under particular sowing environments (Nasim et al., 2010). Cotton cultivars are highly responsive to specific environmental conditions including the day length, specific humidity, temperature and rainfall (Shah et al., 2010). They behave differently to their surrounding environments regarding yield, fiber properties and disease incidence (Moser et al., 2000). Therefore, selection of suitable, aggressive and resistant crop species are the agronomic approaches employed to take yield advantages under varied environmental conditions (Devita et al., 2017). In the changing climatic conditions; selection of superior cotton genotypes according to the varied sowing environment is the dire need of the time. Therefore, two years study was conducted to match suitable time of sowing for Bt cotton genotypes in order to get the higher yield and quality.

\section{Materials and Methods}

\section{Experimental site and soil}

Two years field study was carried out at cotton research institute, Multan. The soil samples from different parts of soil were collected and analyzed to determine different soil properties by following the methods of Homer and Pratt (1961). The soil was silt loam (sand 29\%; silt 53\%; clay 18\%) with EC $1.8 \mathrm{dSm}^{-1}, \mathrm{pH} 8.1$, soil organic matter (0.64\%), available nitrogen total phosphorus and exchangeable potassium were $0.04 \%$, $7.8 \mathrm{mg} \mathrm{kg}^{-1} 169 \mathrm{mg} \mathrm{kg}^{-1}$ respectively. The prevailed weather conditions during the study period are presented in Table 1.

\section{Planting material and experimental details}

Four cotton genotypes IUB-13, MNH-1016, MNH1020 and MNH-1026 were sown at eight different sowing times viz $1^{\text {st }}$ March, $16^{\text {th }}$ March, $1^{\text {st }}$ April, $16^{\text {th }}$ April, $1^{\text {st }}$ May, $16^{\text {th }}$ May, $1^{\text {st }}$ June and $16^{\text {th }}$ June. MNH-1016, MNH-1020 and MNH-1026 were Bt cultivars of cotton research institute, Multan whereas seeds of Bt cultivar IUB-13 were collected from Islamia University, Bahawalpur. The experiment was performed in randomized complete block design with split plot arrangement and was repeated thrice. For both years net plot dimensions were $3 \times 10 \mathrm{~m}$.

\section{Crophusbandry}

Two cultivations followed by one rotavation were made for soil preparation. After that one laser land leveling followed by two cultivations were done for seed bed preparation. Beds and furrows each of 2.5 feet width were made by tractor mounted bed planter. Delinted cotton seeds were manually sown with row and plant space of 75 and $30 \mathrm{~cm}$ respectively. For successful stand establishment re-irrigation after 3 days was done in the furrows and then subsequent irrigations were applied at 7-21 days interval depending upon weather conditions and crop requirement un-till crop maturity. Phosphorus and potassium were applied as basal dose while nitrogen was applied in three splits i.e. at sowing, flowering and boll formation. Thinning at four leaf stage was done to get the desired and healthy plant population. Insect population was 
maintained under economic threshold level through recommended insecticides.

\section{Observations}

\section{Growth and yield parameters}

In each plot, ten plants were marked branches and nodes were counted and averaged. At maturity plant height was determined from tagged plants with meter rod and balls/plant were counted and averaged. Randomly selected 25 opened bolls were picked, weighed and obtained weight was divided with 25 to get the boll weight in grams. Each plot was manually picked three times, weighed and added together along with the weight of 25 opened bolls to determine the seed cotton yield and later on converted into $\mathrm{tha}^{-1}$.

\section{Crop development}

Crop phenological parameter such as time to squaring, flowering and boll opening were determined by daily visual observation of tagged plants in each plot. Each phenological stage was considered when $50 \%$ of the tagged plants attained that stage.

\section{Cotton leaf curl virus infestation}

Fortnight data of cotton leaf curl virus was taken from infested plants showing disease symptoms. Leaves with small and main vein thickness, curling and small 'enation' were considered as an infected and percentage of infected plants was calculated by following methods of Akhtar et al. (2010).

\section{Quality parameters}

Seed cotton samples were sun dried, weighed and ginning was done with the ginning machine. The lint of each collected sample was weighed and ginning out turn (a ratio of lint to seed cotton yield) was determined in percentage. A sub sample of lint (50 g) was taken for determining the micronaire, staple length and fiber strength. High volume instrument spectrum-1 (HVI) was used for determining these physical fiber properties.

\section{Statistical analysis}

The data on different collected traits were analyzed by ANNOVA and differences amid the treatments were compared with least significant difference test at 5\% probability level (Steel et al., 1997).

\section{Results and Discussion}

\section{Growth}

Plant growth parameters were significantly affected by different sowing dates. During first year, maximum plant height $(153.6 \mathrm{~cm})$ and nodes per plant (47.19) were recorded when cotton was sown on $1^{\text {st }}$ April that was statistically same with $16^{\text {th }}$ March sown cotton (Table 2). Minimum plant height $(105.4 \mathrm{~cm})$ and nodes per plant were recorded in crop sown on $16^{\text {th }}$ June sowing (Table 2). Among cotton genotypes, MNH-1026 had highest height and nodes per plant whereas plants of IUB-13 had lowest height and nodes per plant during first year (Table 2). During second year, maximum plant height was noted in genotype MNH-1026 when sown on $1^{\text {st }}$ May and highest nodes per plant were recorded in same genotype in $1^{\text {st }}$ and $16^{\text {th }}$ March sowing date that was statistically same with $1^{\text {st }}$ April, $16^{\text {th }}$ April, $1^{\text {st }}$ May and $16^{\text {th }}$ May sowing date with MNH-1026. Moreover, minimum plant height and nodes per plant were recorded in cotton genotype $\mathrm{MNH}-1016$ when sown at $16^{\text {th }}$ June during second year (Table 2).

Table 1: Weather data of the experimental site during cotton growing seasons 2018 and 2019.

\begin{tabular}{|c|c|c|c|c|c|c|c|c|c|c|}
\hline \multirow[t]{3}{*}{ Months } & \multirow{2}{*}{\multicolumn{2}{|c|}{ Rainfall (mm) }} & \multirow{2}{*}{\multicolumn{2}{|c|}{$\begin{array}{l}\text { Relative } \\
\text { humidity (\%) }\end{array}$}} & \multicolumn{6}{|c|}{ Temperature $\left({ }^{\circ} \mathrm{C}\right)$} \\
\hline & & & & & \multicolumn{2}{|c|}{ Mean maximum } & \multicolumn{2}{|c|}{ Mean minimum } & \multicolumn{2}{|c|}{ Mean } \\
\hline & 2018 & 2019 & 2018 & 2019 & 2018 & 2019 & 2018 & 2019 & 2018 & 2019 \\
\hline March & 0 & 24 & 75.9 & 78.1 & 31.29 & 26.55 & 16.87 & 14.35 & 24.08 & 20.45 \\
\hline April & 8 & 17 & 59.7 & 69.9 & 36.80 & 35.90 & 22.37 & 21.80 & 29.58 & 28.85 \\
\hline May & 4 & 13 & 45.1 & 52.1 & 40.81 & 40.55 & 26.65 & 25.06 & 33.73 & 32.81 \\
\hline June & 4 & 55 & 48.2 & 39.5 & 41.20 & 43.13 & 29.03 & 29.30 & 35.12 & 36.22 \\
\hline July & 6 & 21 & 68.6 & 59 & 38.58 & 39.52 & 29.48 & 29.97 & 34.03 & 34.74 \\
\hline August & 3 & 46 & 71.8 & 71.8 & 37.16 & 38.00 & 28.94 & 28.45 & 33.05 & 33.23 \\
\hline September & 0 & 28 & 64.8 & 67.9 & 36.33 & 38.33 & 26.83 & 28.80 & 31.58 & 33.57 \\
\hline October & 0 & 38 & 68 & 74.6 & 33.97 & 33.45 & 21.16 & 20.52 & 27.56 & 26.98 \\
\hline
\end{tabular}

Source: Cotton Research Institute, Multan, Pakistan. 


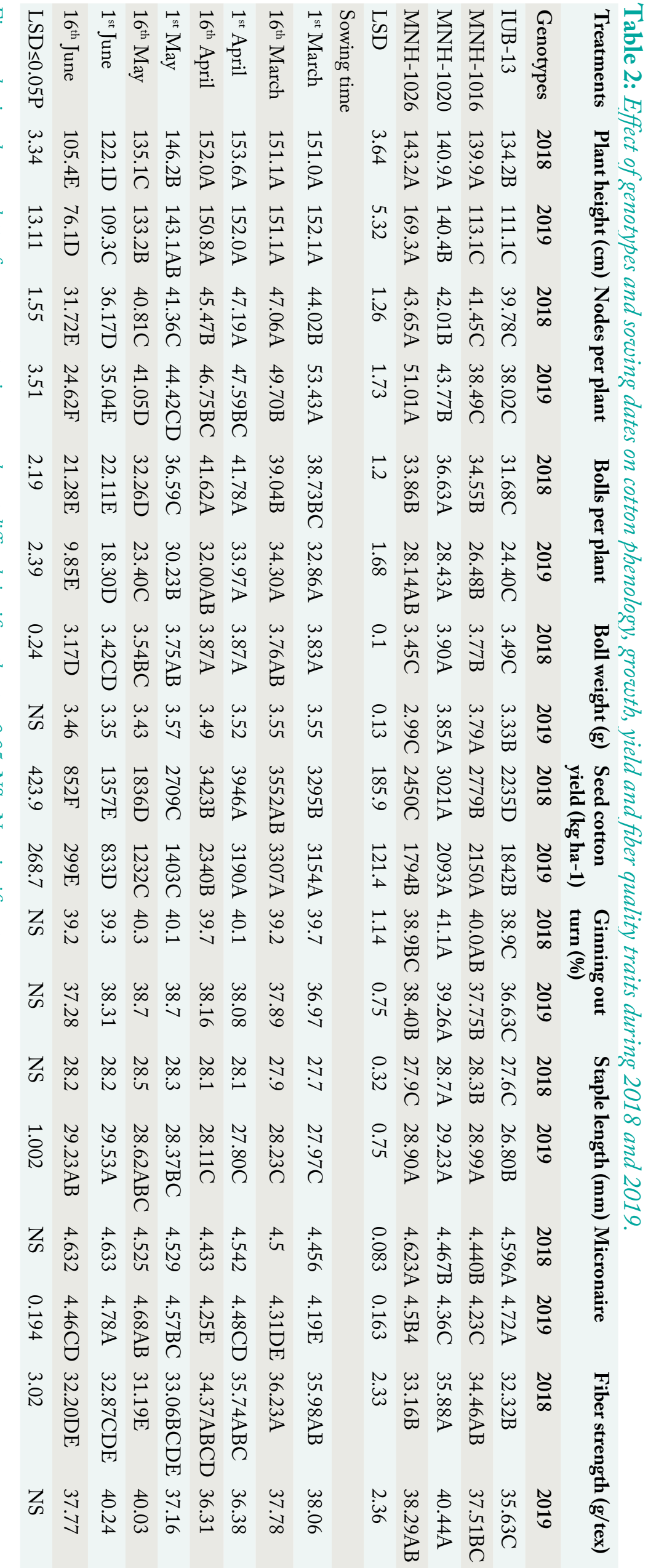


Crop development

Plant mapping data during first year reveal that early sown cotton (March and April) had highest number of squares during the month of July and August whereas late sown cotton (May and June) had highest squares during the month of August and September (Figure 1). However, during second year, early sown cotton had highest squares during August and late sown had during September. Among cotton genotypes during first year highest squares were recorded in the month of August for all genotypes but highest were recorded in IUB-13 followed by MNH-1020. MNH-1020 also had highest squares during July when compared with other genotypes for highest squares during July (Figure 1). Phenological data of crop development verified that during first year lowest time for squaring was noted in MNH-1016 sown on $1^{\text {st }}$ April that was statistically same with the same sowing date with varieties IUB-13 and MNH-1020. During second year, lowest squaring time and boll opening time was recorded in $\mathrm{MNH}-1020$ and highest was noticed in MNH-1026 sown $1^{\text {st }}$ May (Table 3).

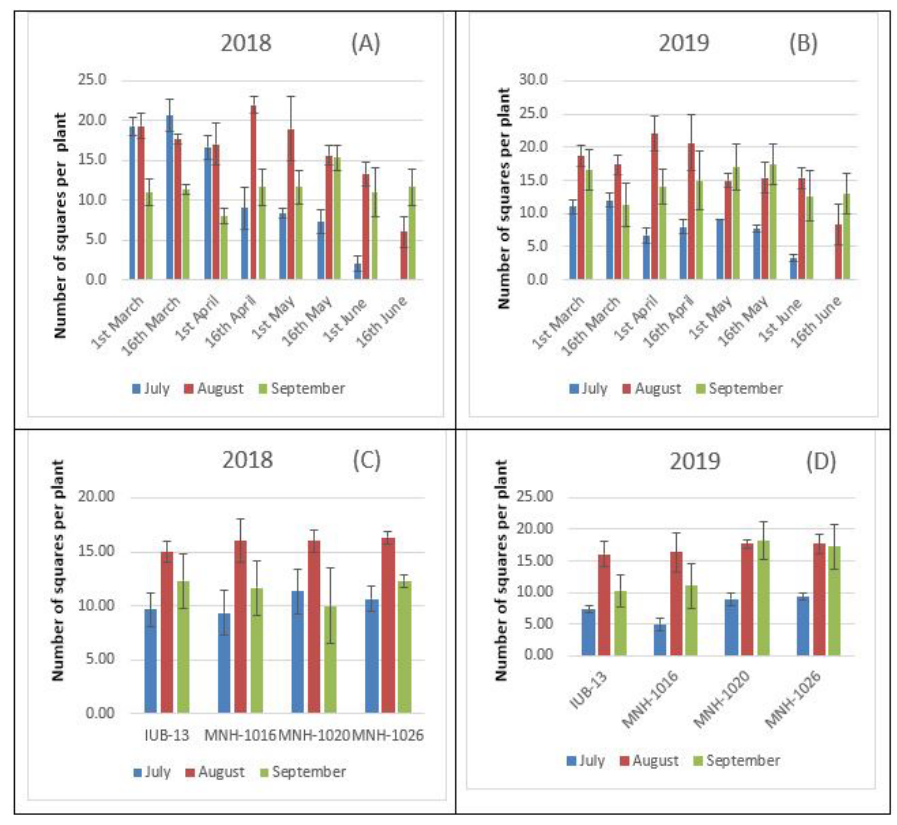

Figure 1: Effect of sowing time on number of squares per plant in new cotton genotypes during 2018 and 2019.

\section{Yield and yield attributes}

Cotton genotypes and sowing dates had significant differences for bolls/plant and boll weight in both studied years (Table 2). For sowing dates, highest bolls per plant and boll weight were recorded in 1st April sown cotton that was statistically similar to $16^{\text {th }}$ April sowing during first year. Among cotton genotypes highest bolls/plant (36.63) and boll weight $(3.90 \mathrm{~g})$ were recorded in cotton genotype MNH-
1020 during both years and lowest bolls were noticed in IUB-13 and boll weight was recorded in MNH1026 during both years (Table 2). In first year of study, highest seed cotton yield was obtained when cotton was planted on $1^{\text {st }}$ April that was statistically similar to seed cotton yield obtained from $16^{\text {th }}$ March sowing. During second year, $16^{\text {th }}$ March sown cotton had highest seed cotton yield that was similar to seed cotton yield obtained from $1^{\text {st }}$ March and $1^{\text {st }}$ April sowing date. Among cotton cultivars, MNH-1020 produced highest seed cotton yield during first year and MNH-1016 during second year, however, it was statistically similar to seed cotton yield obtained from MNH-1020 during second year. Lowest seed cotton yield was recorded in IUB-13 during first year and but statistically similar to IUB-13 during second year (Table 2).

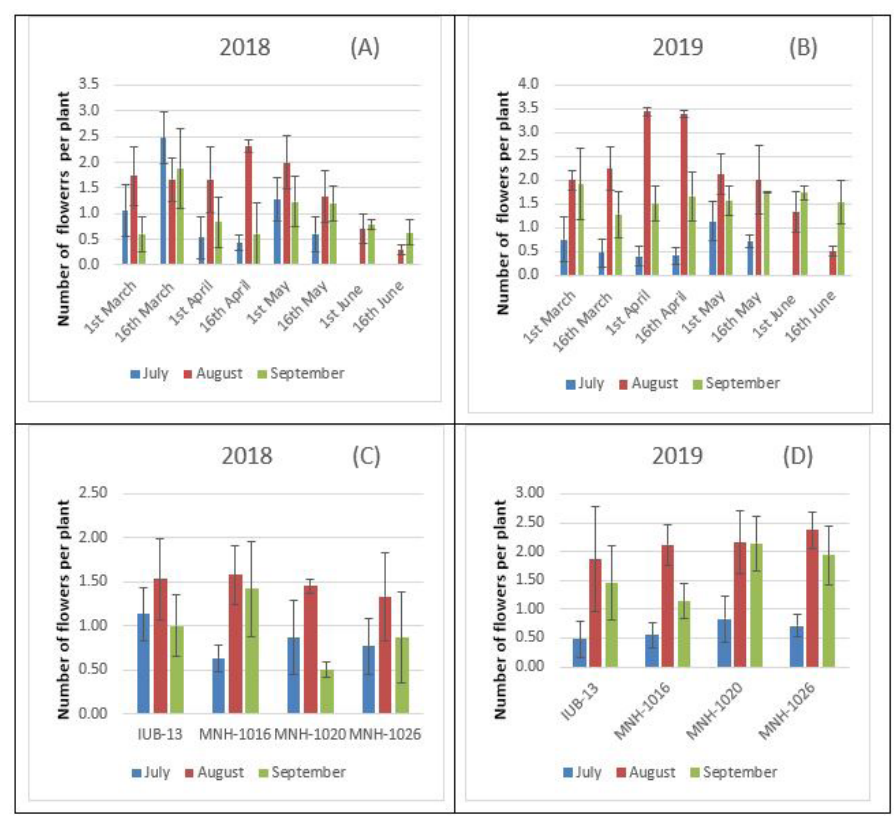

Figure 2: Effect of sowing time on number of flowers per plant in new cotton genotypes during 2018 and 2019.

\section{Cotton leaf curl virus infestation (\%)}

Genotype and sowing environment interaction had significant impact on the infestation of cotton leaf curl virus during both years. Graphical representation of cotton leaf curl virus showed that during first year early sown cotton (March-April) had lowest virus infection during the month of July whereas late sown cotton faced early virus attack during the month of July limiting plant growth and development. During second year early sown cotton was least infected by virus during July, August and September compared to late sowing (Figure 4). During first year, lowest virus infestation was recorded in cotton cultivar MNH1020 planted on $1^{\text {st }}$ March that was statistically 
similar to the $1^{\text {st }}$ April with same variety. Highest virus infestation was recorded in cotton cultivar IUB13 sown on $16^{\text {th }}$ June 2019. During second year, cotton cultivar MNH-1020 planted on $16^{\text {th }}$ April was least effected with virus whereas same variety sown on $1^{\text {st }}$ May exhibited greatest virus infestation (Figure 4).

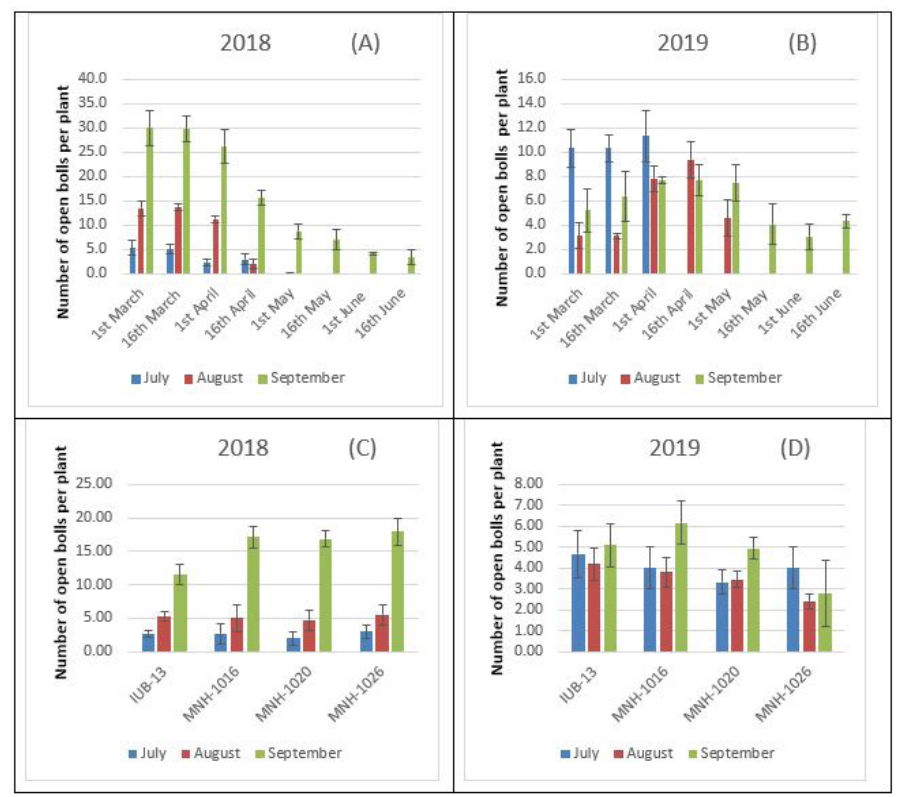

Figure 3: Effect of sowing time on number of open bolls per plant in new cotton genotypes during 2018 and 2019.

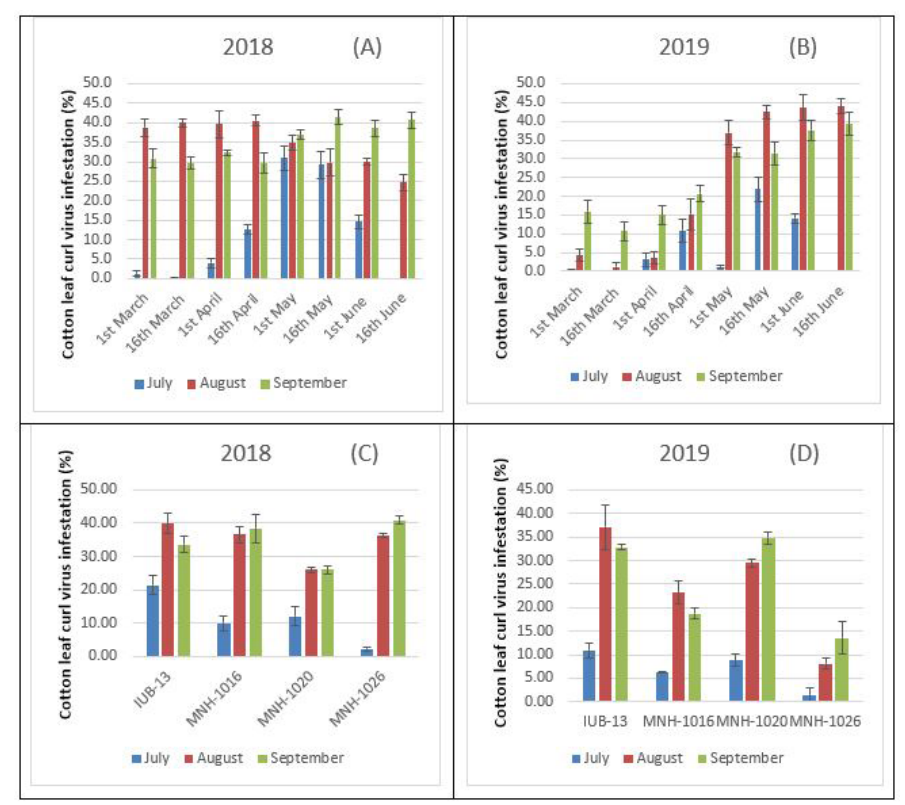

Figure 4: Effect of sowing time on cotton leaf curl virus infestation (\%) in new cotton genotypes during 2018 and 2019.

\section{Cotton fiber quality}

MNH-1020 had highest ginning out turn $(41.1,39.3)$ and staple length $(28.7,29.23 \mathrm{~cm})$ whereas IUB-13 had lowest GOT $(38.90,36.63 \%)$ and staple length $(27.6,26.80 \mathrm{~cm})$ during both years. During second year, cotton cultivar MNH-1026 had highest staple length $(30.90 \mathrm{~cm})$ when planted on $16^{\text {th }}$ June. Lowest staple length was attained in cotton cultivar IUB13 with $16^{\text {th }}$ April sowing statistically similar with $1^{\text {st }}$ April sowing (Table 4). Lowest Mike maximum value was recorded in cotton genotype $\mathrm{MNH}-1016$ that was statistically same with MNH-1020 during both years. For sowing dates $1^{\text {st }}$ March sowing had least mike similar with $16^{\text {th }}$ March and $16^{\text {th }}$ April. Cotton sown on $1^{\text {st }}$ June had greatest Mike (Table 2). Fiber of $\mathrm{MNH}-1020$ had greatest strength during both years. $16^{\text {th }}$ March sown cotton had greatest fiber strength whereas $16^{\text {th }}$ May sown cotton has lowest fiber strength (Table 2).

Results of two years field trials confirmed that along with genetic makeup varied sowing environments also had significant impact on the growth and quality traits and cotton yield. Genotypes with diverse background vary in terms of growth and development (Bange and Milroy, 2004). Plant height and nodes per plant were highest in MNH-1026 because of genetic difference (Boquet and Clawson, 2009) but sowing window from $1^{\text {st }}$ March to $30^{\text {th }}$ April (Early sown cotton) also had highest expression of these growth parameters (Table 2) owing to environmental conditions prevailed during growth of crop. Advance sowing had early climatic support of higher sunshine hours, effective rainfall and total water used by crop (Table 1) that caused better crop growth (Patil et al., 2009).

Data regarding cotton leaf curl virus infestation revealed that during August and September 2018 cotton leaf curl virus infestation was highest compared to 2019 (Figure 4). High rainfall during second year may be the reason of low attack CLCV during second year. However, growing of resistant genotype with advanced sowing date is vital to reduce the attack of cotton leaf curl (Karavina et al., 2012). Low infestation of CLCV on cotton genotype MNH-1020 when planted early increased seed cotton yield (Table 2) whereas high infestation of cotton leaf curl virus on late planted IUB-13 decreased its yield (Table 2). Due to enhanced growth, early sown cotton (MarchApril) withstands the attack of leaf curl virus (Pedigo 2004). Moreover, early plantation escaped from virus stress (Figure 4) during peak flowering time (Figure 2) while late planted crop hitted very earlier by CLCV and infestation becomes severe during peak flowering period resulting in reduction in yield (Gormus and Yucel, 2002). 
Table 3: Interactive effect of sowing date and cultivar on time to squaring, flowering and boll opening in cotton.

\section{Treatments}

\begin{tabular}{|c|c|c|c|c|c|c|c|}
\hline & & 2018 & 2019 & 2018 & 2019 & 2018 & 2019 \\
\hline \multirow[t]{4}{*}{$1^{\text {st }}$ March } & IUB-13 & 39.00ghi & 44.00defg & $54.00 \mathrm{jk}$ & $64.67 \mathrm{bcd}$ & 88.33hijk & $99.00 \mathrm{efgh}$ \\
\hline & MNH-1016 & $41.00 \mathrm{ef}$ & $48.00 \mathrm{a}$ & $57.00 \mathrm{f}$ & $68.33 a$ & 88.00hijk & $101.67 \mathrm{cdef}$ \\
\hline & MNH-1020 & $39.33 \mathrm{gh}$ & 46.67ab & $57.00 \mathrm{f}$ & $67.67 \mathrm{a}$ & $87.33 \mathrm{ijk}$ & $102.00 \mathrm{cde}$ \\
\hline & MNH-1026 & 38.00hijk & $46.22 \mathrm{abc}$ & 54.33jk & $67.11 \mathrm{ab}$ & $86.33 \mathrm{k}$ & $101.83 \mathrm{cde}$ \\
\hline \multirow[t]{4}{*}{$16^{\text {th }}$ March } & IUB-13 & $39.33 \mathrm{gh}$ & 42.67fghi & 55.00hijk & $62.67 \mathrm{def}$ & $88.33 \mathrm{de}$ & $94.67 \mathrm{ijk}$ \\
\hline & MNH-1016 & $37.00 \mathrm{kl}$ & 41.67hi & 55.33ghij & $61.00 \mathrm{fg}$ & 88.00hijk & 97.33ghij \\
\hline & MNH-1020 & $41.67 \mathrm{de}$ & 42.00ghi & $56.33 \mathrm{fgh}$ & $64.67 \mathrm{bcd}$ & $88.33 \mathrm{hijk}$ & $94.67 \mathrm{ijk}$ \\
\hline & MNH-1026 & 38.67ghij & 42.11fghi & $54.67 \mathrm{ijk}$ & $62.67 \mathrm{def}$ & 88.67ghijk & 95.44hijk \\
\hline \multirow[t]{4}{*}{$1^{\text {st }}$ April } & IUB-13 & $34.67 \mathrm{mn}$ & $40.67 \mathrm{ij}$ & $47.67 \mathrm{o}$ & 57.00hi & $87.67 \mathrm{ijk}$ & $93.67 \mathrm{jk}$ \\
\hline & MNH-1016 & $33.33 n$ & 42.00ghi & $53.67 \mathrm{kl}$ & $59.00 \mathrm{gh}$ & $87.00 \mathrm{ijk}$ & 98.00fghi \\
\hline & MNH-1020 & $34.67 \mathrm{mn}$ & 41.00ij & $50.33 n$ & $58.67 \mathrm{gh}$ & $87.00 \mathrm{ijk}$ & $95.00 \mathrm{ijk}$ \\
\hline & MNH-1026 & $35.00 \mathrm{~m}$ & 41.22hi & $50.33 n$ & $58.22 \mathrm{~h}$ & $87.00 \mathrm{ijk}$ & 95.56hijk \\
\hline \multirow[t]{4}{*}{$16^{\text {th }}$ April } & IUB-13 & $34.67 \mathrm{mn}$ & $38.33 \mathrm{k}$ & $52.00 \mathrm{~m}$ & $57.00 \mathrm{hi}$ & $86.67 \mathrm{jk}$ & $95.00 \mathrm{ijk}$ \\
\hline & MNH-1016 & 37.67ijk & $37.33 \mathrm{kl}$ & 55.33ghij & 57.00hi & $86.33 \mathrm{k}$ & $95.00 \mathrm{ijk}$ \\
\hline & MNH-1020 & $40.00 \mathrm{fg}$ & $37.67 \mathrm{k}$ & $52.331 \mathrm{~m}$ & $54.33 \mathrm{j}$ & $86.33 \mathrm{k}$ & $91.67 \mathrm{kl}$ \\
\hline & MNH-1026 & $35.33 \mathrm{~m}$ & $35.671 \mathrm{~m}$ & $52.00 \mathrm{~m}$ & $55.33 \mathrm{ij}$ & $86.33 \mathrm{k}$ & $103.33 \mathrm{~cd}$ \\
\hline \multirow[t]{4}{*}{$1^{\text {st }}$ May } & IUB-13 & $46.00 \mathrm{bc}$ & $37.00 \mathrm{kl}$ & $62.67 \mathrm{c}$ & $51.00 \mathrm{k}$ & $87.67 \mathrm{ijk}$ & $83.00 \mathrm{~m}$ \\
\hline & MNH-1016 & $45.67 c$ & $40.67 \mathrm{ij}$ & $64.33 \mathrm{~b}$ & $55.00 \mathrm{ij}$ & 91.00defg & 89.671 \\
\hline & MNH-1020 & $47.00 \mathrm{abc}$ & $35.00 \mathrm{~m}$ & $67.00 \mathrm{a}$ & $51.67 \mathrm{k}$ & 89.00fghij & $81.00 \mathrm{~m}$ \\
\hline & MNH-1026 & $46.33 b c$ & $38.33 \mathrm{k}$ & $66.33 a$ & $55.67 \mathrm{ij}$ & 90.33efgh & $114.33 a$ \\
\hline \multirow[t]{4}{*}{$16^{\text {th }}$ May } & IUB-13 & $47.00 \mathrm{abc}$ & 46.67ab & 57.33ef & 66.67ab & $86.33 \mathrm{k}$ & 98.00fghi \\
\hline & MNH-1016 & 47.33ab & 43.33efgh & $62.00 \mathrm{c}$ & $62.67 \mathrm{def}$ & 88.33hijk & $100.67 \mathrm{defg}$ \\
\hline & MNH-1020 & $47.00 \mathrm{abc}$ & 42.33fghi & $60.33 d$ & $61.67 \mathrm{ef}$ & 89.33fghi & $101.33 \mathrm{def}$ \\
\hline & MNH-1026 & $48.33 a$ & $39.00 \mathrm{jk}$ & $62.67 c$ & 66.33ab & $93.00 \mathrm{~cd}$ & $107.33 b$ \\
\hline \multirow[t]{4}{*}{$1^{\text {st }}$ June } & IUB-13 & $41.67 \mathrm{de}$ & 43.33efgh & $58.67 \mathrm{e}$ & 56.67hij & $92.00 \mathrm{de}$ & $96.00 \mathrm{hij}$ \\
\hline & MNH-1016 & $43.00 \mathrm{~d}$ & 42.67fghi & $60.33 d$ & $62.00 \mathrm{ef}$ & 91.33def & 97.33ghij \\
\hline & MNH-1020 & $43.00 \mathrm{~d}$ & $44.33 \mathrm{cdef}$ & $61.33 \mathrm{~cd}$ & 56.67hij & 89.33fghi & 95.67hij \\
\hline & MNH-1026 & $43.00 \mathrm{~d}$ & $45.67 \mathrm{bcd}$ & $60.33 d$ & 63.67cde & 88.67ghijk & $102.67 \mathrm{cde}$ \\
\hline \multirow[t]{4}{*}{$16^{\text {th }}$ June } & IUB-13 & $35.671 \mathrm{~m}$ & $46.67 \mathrm{ab}$ & $55.33 \mathrm{ghij}$ & $65.00 \mathrm{bcd}$ & $93.33 \mathrm{~cd}$ & $102.67 \mathrm{cde}$ \\
\hline & MNH-1016 & $35.33 \mathrm{~m}$ & $47.00 \mathrm{ab}$ & $56.67 \mathrm{fg}$ & $68.00 \mathrm{a}$ & $95.00 \mathrm{bc}$ & $103.00 \mathrm{~cd}$ \\
\hline & MNH-1020 & $37.33 \mathrm{jk}$ & $45.33 \mathrm{bcde}$ & 56.00fghi & $66.00 \mathrm{abc}$ & $97.67 \mathrm{a}$ & 102.67 cdee \\
\hline & MNH-1026 & $40.00 \mathrm{fg}$ & 46.33abcd & 56.33fgh & $58.33 \mathrm{~h}$ & $96.67 \mathrm{ab}$ & $105.33 b c$ \\
\hline $\mathrm{LSD} \leq 0.05 \mathrm{P}$ & & 1.56 & 1.97 & 1.42 & 2.55 & 2.45 & 3.78 \\
\hline
\end{tabular}

Time to flowering (Days) Time to boll opening (Days)

Time to squaring (Days)

2019

44.00defg

$46.67 \mathrm{ab}$

$46.22 \mathrm{abc}$

41.67hi

$40.67 \mathrm{ij}$

$41.00 \mathrm{ij}$

$41.22 \mathrm{hi}$

$37.33 \mathrm{kl}$
2.55
2.45

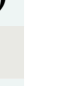

Figures sharing the same letter for a parameter in a year do not differed significantly at p $\leq 0.05$, NS= Non significant.

Yield determining parameters such as bolls/plant and boll weight were greater during first year because of decrease in monthly mean temperature during July, August and September (peak flowering period) and low rainfall compared to second years (Table 1). An increase in temperature had profound effect on flower shedding and boll retention (Fisher, 1975). However, cotton plants sown early have the ability to compensate better by producing new floral parts and convert them into yield traits compared to late planted plants (Table 2). Because of extended growth period due to early planting, plants received additional soil moisture and nutrients which favored the more balls to mature (Huang and Ji, 2016). Early planted cotton had improved boll size (Table 2) owing to accretion of more assimilates and prolonged period for ball development as well as maturity (Pettigrew, 2002; Nuti et al., 2006). Whereas late-planted cotton had more flowers and bolls later in the growing season (Figure 2) with low temperature (Table 1) lengthened the period from sowing to boll opening (Table 3) which delayed maturity and reduced yield (Elayan $e t$ al., 2015; Wanga et al., 2016). 
Table 4: Interactive effect of sowing date and cultivars on plant height, number of nodes, bolls per plant, cotton leaf curl virus infestation and staple length in cotton.

\section{Treatments}

\begin{tabular}{|c|c|c|c|c|c|}
\hline & & 2018 & 2019 & 2018 & 2019 \\
\hline \multirow[t]{4}{*}{$1^{\text {st }}$ March } & IUB-13 & 147.3 & $141.1 \mathrm{~h}-\mathrm{j}$ & 42.37 & $52.23 \mathrm{~b}-\mathrm{d}$ \\
\hline & MNH-1016 & 150.7 & $128.9 \mathrm{jk}$ & 42.63 & $49.97 c-f$ \\
\hline & MNH-1020 & 151.9 & $158.6 \mathrm{~d}-\mathrm{g}$ & 44.47 & $52.87 \mathrm{~b}-\mathrm{d}$ \\
\hline & MNH-1026 & 154 & $179.9 \mathrm{bc}$ & 46.6 & $58.63 a$ \\
\hline \multirow[t]{4}{*}{$16^{\text {th }}$ March } & IUB-13 & 147.5 & $132.4 \mathrm{i}-\mathrm{k}$ & 45.83 & $46.33 \mathrm{e}-\mathrm{g}$ \\
\hline & MNH-1016 & 150.5 & $142.4 \mathrm{~g}-\mathrm{j}$ & 46.8 & $48.87 \mathrm{~d}-\mathrm{f}$ \\
\hline & MNH-1020 & 151.8 & $155.2 \mathrm{e}-\mathrm{h}$ & 47.03 & $44.97 f-h$ \\
\hline & MNH-1026 & 154.7 & $174.3 \mathrm{~cd}$ & 48.57 & $58.63 a$ \\
\hline \multirow[t]{4}{*}{$1^{\text {st }}$ April } & IUB-13 & 148.1 & $141.7 \mathrm{~g}-\mathrm{j}$ & 44.07 & $44.48 \mathrm{f}-\mathrm{h}$ \\
\hline & MNH-1016 & 154.1 & $125.0 \mathrm{j}-1$ & 47.27 & $39.85 \mathrm{~h}-\mathrm{j}$ \\
\hline & MNH-1020 & 155.5 & $167.2 \mathrm{c}-\mathrm{e}$ & 47.6 & $52.13 \mathrm{~b}-\mathrm{d}$ \\
\hline & MNH-1026 & 156.5 & $173.9 \mathrm{~cd}$ & 49.83 & $53.90 \mathrm{a}-\mathrm{d}$ \\
\hline \multirow[t]{4}{*}{$16^{\text {th }}$ April } & IUB-13 & 144.4 & $126.9 \mathrm{j}-1$ & 42.87 & $41.55 \mathrm{gh}$ \\
\hline & MNH-1016 & 151.1 & $134.9 \mathrm{i}-\mathrm{k}$ & 45.27 & 42.14gh \\
\hline & MNH-1020 & 154.5 & $165.9 c-f$ & 45.93 & $51.23 \mathrm{~b}-\mathrm{e}$ \\
\hline & MNH-1026 & 158 & $175.6 \mathrm{~cd}$ & 47.8 & $52.06 \mathrm{~b}-\mathrm{d}$ \\
\hline \multirow[t]{4}{*}{$1^{\text {st }}$ May } & IUB-13 & 143.6 & $104.7 \mathrm{~m}-\mathrm{o}$ & 39.1 & $35.15 \mathrm{j}-1$ \\
\hline & MNH-1016 & 146 & $118.8 \mathrm{k}-\mathrm{m}$ & 41.7 & 40.67hi \\
\hline & MNH-1020 & 147.3 & $148.6 f-i$ & 41.63 & $45.30 f-h$ \\
\hline & MNH-1026 & 147.8 & $200.1 \mathrm{a}$ & 43 & $56.56 \mathrm{ab}$ \\
\hline \multirow[t]{4}{*}{$16^{\text {th }}$ May } & IUB-13 & 123.1 & $90.2 \mathrm{op}$ & 38.5 & $31.75 \mathrm{k}-\mathrm{m}$ \\
\hline & MNH-1016 & 137 & $110.01-\mathrm{n}$ & 39.77 & $35.96 \mathrm{i}-\mathrm{k}$ \\
\hline & MNH-1020 & 139.3 & $135.5 \mathrm{i}-\mathrm{k}$ & 42.23 & $41.55 \mathrm{gh}$ \\
\hline & MNH-1026 & 141.1 & $197.0 \mathrm{ab}$ & 42.73 & $54.93 a-c$ \\
\hline \multirow[t]{4}{*}{$1^{\text {st }}$ June } & IUB-13 & 119.5 & 87.3op & 35.1 & $30.421 \mathrm{~m}$ \\
\hline & MNH-1016 & 122.5 & $82.9 p q$ & 36.57 & $29.56 \mathrm{~m}$ \\
\hline & MNH-1020 & 120.5 & $109.51-\mathrm{n}$ & 34.83 & $35.05 \mathrm{j}-1$ \\
\hline & MNH-1026 & 125.8 & $157.4 d-h$ & 38.17 & $45.15 f-h$ \\
\hline \multirow[t]{4}{*}{$16^{\text {th }}$ June } & IUB-13 & 99.9 & $64.5 \mathrm{qr}$ & 30.43 & 22.23 no \\
\hline & MNH-1016 & 107.6 & $61.8 \mathrm{r}$ & 31.57 & 20.91o \\
\hline & MNH-1020 & 106.4 & $82.4 p q$ & 32.37 & $27.11 \mathrm{mn}$ \\
\hline & MNH-1026 & 108 & $95.7 n-p$ & 32.5 & $28.22 \mathrm{~m}$ \\
\hline $\mathrm{LSD} \leq 0.05 \mathrm{P}$ & & NS & 18.473 & NS & 5.49 \\
\hline
\end{tabular}

Bolls per plant Cotton leaf curl virus Staple length

infestation (\%) (mm)

$\begin{array}{lllll}2018 & 2019 & 2018 & 2019 & 2018\end{array}$

$37.27 \quad 31.50 b-d \quad 44.67 \mathrm{e}-\mathrm{h} \quad 28.33 \mathrm{f}-\mathrm{h} \quad 27.2 \quad 26.52 \mathrm{j}$

$38.27 \quad 32.90 \mathrm{a}-\mathrm{d} \quad 41.33 \mathrm{f}-\mathrm{j} \quad 11.33 \mathrm{k}-\mathrm{m} \quad 27.8 \quad 28.25 \mathrm{~d}-\mathrm{i}$

$\begin{array}{llllll}40.57 & 35.47 \mathrm{ab} & 25.001 & 14.67 \mathrm{kl} & 28.3 & 29.48 \mathrm{a}-\mathrm{d}\end{array}$

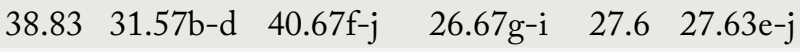

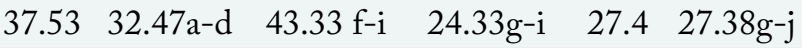

$38.7 \quad 35.27 \mathrm{a}-\mathrm{d} \quad 40.00 \mathrm{f}-\mathrm{j} \quad 9.67 \mathrm{k}-\mathrm{m} \quad 28 \quad 28.42 \mathrm{~d}-\mathrm{h}$

$\begin{array}{llllll}41.07 & 36.53 \mathrm{a} & 35.67 \mathrm{i}-\mathrm{k} & 7.33 \mathrm{mn} & 28.4 & 29.82 \mathrm{abc}\end{array}$

$38.87 \quad 32.93 \mathrm{a}-\mathrm{d} \quad 40.00 \mathrm{f}-\mathrm{j} \quad 22.00 \mathrm{~h}-\mathrm{j} \quad 27.8 \quad 27.30 \mathrm{~g}-\mathrm{j}$

$40.17 \quad 33.00 \mathrm{a}-\mathrm{d} \quad 43.00 \mathrm{f}-\mathrm{j} \quad 21.33 \mathrm{ij} \quad 27.3 \quad 26.37 \mathrm{j}$

$\begin{array}{llllll}41.83 & 34.00 \mathrm{a}-\mathrm{c} & 35.67 \mathrm{jk} & 8.331-\mathrm{n} & 28.6 & 28.67 \mathrm{c}-\mathrm{g}\end{array}$

$\begin{array}{llllll}43.47 & 35.77 \mathrm{ab} & 25.671 & 34.00 \mathrm{~d}-\mathrm{f} & 28.8 & 28.62 \mathrm{c}-\mathrm{g}\end{array}$

$41.67 \quad 33.10 \mathrm{a}-\mathrm{d} \quad 51.33 \mathrm{cde} \quad 38.33 \mathrm{~cd} \quad 27.6 \quad 27.57 \mathrm{f}-\mathrm{j}$

$40.07 \quad 29.80 \mathrm{c}-\mathrm{e} \quad 37.73 \mathrm{~h}-\mathrm{k} \quad 37.33 \mathrm{~cd} \quad 27.6 \quad 26.13 \mathrm{j}$

$42.07 \quad 32.20 \mathrm{a}-\mathrm{d} \quad 37.67 \mathrm{~h}-\mathrm{k} \quad 6.67 \mathrm{mn} \quad 28.2 \quad 28.23 \mathrm{~d}-\mathrm{i}$

$43.13 \quad 36.13 \mathrm{ab} \quad 35.43 \mathrm{jk} \quad 3.00 \mathrm{n} \quad 29 \quad 28.58 \mathrm{c}-\mathrm{g}$

$41.2 \quad 29.87 \mathrm{cde} \quad 40.00 \mathrm{f}-\mathrm{j} \quad 40.33 \mathrm{~b}-\mathrm{d} \quad 27.8 \quad 29.50 \mathrm{a}-\mathrm{d}$

$34.2 \quad 28.90 \mathrm{de} \quad 45.67 \mathrm{~d}-\mathrm{g} \quad 40.33 \mathrm{~b}-\mathrm{d} \quad 27.8 \quad 26.90 \mathrm{~h}-\mathrm{j}$

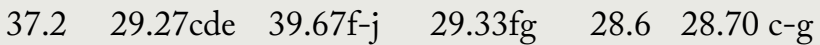

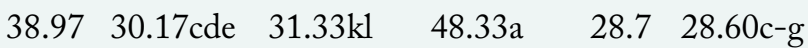

$36 \quad 32.60 \mathrm{a}-\mathrm{d} \quad 38.67 \mathrm{~g}-\mathrm{k} \quad 11.67 \mathrm{k}-\mathrm{m} \quad 28.2 \quad 29.27 \mathrm{~b}-\mathrm{d}$

$\begin{array}{llllll}28.53 & 16.23 \mathrm{~g} & 57.67 \mathrm{bc} & 45.33 \mathrm{ab} & 28.2 & 27.00 \mathrm{~h}-\mathrm{j}\end{array}$

$31.33 \quad 21.80 f \quad 44.67 \mathrm{e}-\mathrm{h} \quad 23.33 \mathrm{~g}-\mathrm{i} \quad 28.7 \quad 29.27 \mathrm{~b}-\mathrm{d}$

$35.1 \quad 26.00 \mathrm{ef} \quad 41.67 \mathrm{f}-\mathrm{j} \quad 34.67 \mathrm{c}-\mathrm{f} \quad 29.1 \quad 29.13 \mathrm{c}-\mathrm{e}$

$34.07 \quad 29.57 \mathrm{c}-\mathrm{e} \quad 46.67 \mathrm{~d}-\mathrm{f} \quad 15.67 \mathrm{jk} \quad 28 \quad 29.07 \mathrm{~b}-\mathrm{f}$

$17.97 \quad 14.57 \mathrm{gh} \quad 59.93 \mathrm{~b} \quad 34.67 \mathrm{c}-\mathrm{f} \quad 27.6 \quad 27.40 \mathrm{~g}-\mathrm{j}$

$24.2 \quad 16.03 \mathrm{~g} \quad 47.07 \mathrm{~d}-\mathrm{f} \quad 34.67 \mathrm{c}-\mathrm{f} \quad 28.1 \quad 30.40 \mathrm{ab}$

$26.37 \quad 16.50 \mathrm{~g} \quad 43.83 \mathrm{e}-\mathrm{h} \quad 46.00 \mathrm{ab} \quad 29 \quad 30.30 \mathrm{ab}$

$19.9 \quad 26.10 \mathrm{ef} \quad 55.73 \mathrm{bc} \quad 29.67 \mathrm{e}-\mathrm{g} \quad 27.9 \quad 30.00 \mathrm{a}-\mathrm{c}$

$\begin{array}{llllll}17.67 & 8.77 \mathrm{i} & 76.00 \mathrm{a} & 40.67 \mathrm{bc} & 27.9 & 26.73 \mathrm{ij}\end{array}$

$22.8 \quad 10.33 \mathrm{hi} \quad 53.00 \mathrm{bcd} \quad 41.00 \mathrm{bc} \quad 28.3 \quad 29.97 \mathrm{a}-\mathrm{c}$

24.33 10.90hi 46.67def $37.33 \mathrm{~cd} \quad 28.5 \quad 29.33 \mathrm{~b}-\mathrm{d}$

$20.33 \quad 9.40 \mathrm{i} \quad 59.00 \mathrm{~b} \quad 36.00 \mathrm{c}-\mathrm{e} \quad 28.1 \quad 30.90 \mathrm{a}$

NS $\quad 4.7432 \quad$ NS $\quad 6.58 \quad$ NS 1.52

Figures sharing the same letter for a parameter in a year do not differed significantly at $p \leq 0.05, N S=$ Non-significant.

Highest ginning out turn, fiber strength as well as staple length in cotton genotype MNH-1020 (Table 2) authenticated that genetic makeup of cultivars plays a major role in the fiber strength and length (Jordan, 2001) and lint index (O'Berry et al., 2009). The quantity of deposited cellulose determines the fiber strength, fineness and maturity (Ramey, 1999). The cultivars having the long molecules of cellulose has the higher fiber strength owing to presence of few breaking points in lint and superior cross linking amid the fibers (Jordan, 2001). Fiber quality mainly influenced by cultivars, whilst management considerations are the secondary one (Bednarz et al., 2005). Ginning out turn was not effected with late or early planting during both years (Table 2). Similar results were noticed by Braunack et al. (2012). However, micronaire value declined in early sowing compared to later sowing (Table 2). Early planting 
avoid decline in macronaire value (Deho et al., 2012). Late planted cotton had more yellow and grey fiber owing to late harvesting which exposes the fiber to different environment conditions not favorable for development of fiber (Duckett et al., 1999). Early sown cotton had highest fiber strength compared to late sown (Table 2). Late planted cotton reached at maturity later in the season and farmers harvest the immature cotton have low fiber strength and poor dye uptake capacity (Bradow and Bauer, 1997).

\section{Conclusions and Recommendations}

For varied sowing dates, increased expression of yield and yield related parameters due to decreased incidence of cotton leaf curl virus disease argued that cotton sowing window should be from $1^{\text {st }}$ March to $16^{\text {th }}$ April for maximum and quality harvest. Among cotton genotypes $\mathrm{MNH}-1020$ has the potential to perform better under arid conditions where low rainfall and high temperatures are the main characteristics.

\section{Novelty Statement}

The rapid climate changes direly need the optimization of agronomic practices to ensure the better crop yield and quality. The sowing time is an important management consideration which plays a significant role in the final cotton yield and quality. However, limited studies are conducted to determine the optimum sowing time for cotton cultivars grown in Agro-Ecological Conditions of Multan. Therefore this study determine the suitable planting time for sowing of different cotton cultivars under Agro-Ecological Conditions of Multan.

\section{Author's Contribution}

Muhammad Iqbal and Muhammad Mahmood Iqbal: Conducted the experiment and wrote the original draft.

Saghir Ahmad, Athar Mahmood, Muhammad Akram, Saeed Ahmad, Ali Raza, Ansar Hussain, Allah Ditta Abid, Qaisar Abbas, Mussarrat Hussain, Muhammad Akram and Muhammad Umair Hassan: Reviewed and edited the article.

Hammad Husnain and Muhammad Shahid: Helped in Data collection.

\section{Conflict of interest}

The authors have declared no conflict of interest.

\section{References}

Akhtar, K.P., S. Haider, M.K.R. Khan, M. Ahmad, N. Sarwar, M.A. Murtaza and M. Aslam. 2010. Evaluation of Gossypium species for resistance to leaf curl burewala virus. Ann. App. Biol., 157: 135-147.

Bange, M.P. and S.P. Milroy. 2004. Growth and dry matter partitioning of diverse cotton genotypes. Field Crops Res., 87: 73-87. https://doi. org/10.1016/j.fcr.2003.09.007

Bednarz, C.W., W.D. Shurley, W.S. Anthony and R.L. Nichols. 2005. Yield, quality, and profitability of cotton produced at varying plant densities. Agron. J. 97, 235-240.

Boquet, D.J. and E.L. Clawson. 2009. Cotton planting date: Yield, seedling survival, and plant growth. Agron. J. 101: 1123-1130. https://doi. org/10.2134/agronj2009.0071

Bradow, J.M. and G.H. Davidonis. 2000. Quantitation of fiber quality and the cotton production- processing interface: A physiologist's perspective. J. Cot. Sci., 4: 34-64.

Bradow, J.M. and P.J. Bauer. 1997. How variety and weather determine yarn properties and dye uptake. Beltwide cotton conf., new orleans, LA. 7-10 Jan. National Cotton Counc. Am. Memphis, TN, pp. 560-564.

Braunack, M.V., M.P. Bange and B.D. Johnston. 2012. Can plating data and cultivar selection improve use efficiency of cotton systems? Field Crop Res., 137:1-11.https://doi.org/10.1016/j. fcr.2012.08.018

Chen, X., D. Min, T.A. Yasir and Y.G. Hu. 2012. Evaluation of 14 morphological, yieldrelated and physiological traits as indicators of drought tolerance in Chinese winter bread wheat revealed by analysis of the membership function value of drought tolerance (MFVD). Field Crops Res., 137:195-201.

Chattha, M.U., I. Khan, M.U. Hassan, M. Nawaz, M.B. Chattha, N.H. Ahamd, M. Usman, M. Kharal and A.U. Khan. 2017. Agronomic appraisal of amaranth accessions under semiarid conditions of Pakistan. Pak. Life Soc. Sci., 15(3): 144-149. https://doi.org/10.1016/ S0378-4290(02)00121-1

Chattha, M.U., M.U. Hassan, I. Khan, M.B. Chattha, M. Aamer, M. Nawaz, S.A. Anjum, U. Ashraf and M. Kharal. 2020. Impact of planting 
methods on biomass production, chemical composition and methane yield of sorghum cultivars. Pak. J. Agric. Sci., 57(1): 43-51.

Deho, Z.A., S. Laghari, S. Abro, S.D. Khanzada and Fakhuruddin. 2012. Effect of sowing dates and picking intervals at boll opening percent, yield and fiber quality of cotton cultivars. Sci. Tech. Dev., 31(3): 288-293.

Devita. P., S.A. Colecchia, I. Pecorella and S. Sai. 2017. Reduced inter-row distance improves yield and competition against weeds in a semidwarf durum wheat variety. Eur. J. Agro. 85: 6977. https://doi.org/10.1016/j.eja.2017.02.003

Duckett, K.E., L. Cheng, T. Sapaletalova, M. Watson and H. Ghorashi. 1999. Color grading of cotton measurement (Part I). In Proc. Beltwide Cotton Conf., Orlando, FL. 3-7 Jan. 1999 p. 645-650.

Elayan, E.D., A.M.A. Sohair, S.D. Abdalla, S.D. Nadia and A.E.F. Wageda. 2015. Effect of delaying planting date on yield, fiber and yarn quality properties in some cultivars and promising crosses of Egyptian cotton. Am. Eur. J. Agric. Environ. Sci., 15(5): 754-763.

Fisher. W.D. 1975. Heat induced sterility in upland cotton. Proceedings of the 27th Cotton Improvement Conference, National Cotton Council, Memphis, Tenn. Pp. 85.

Gormus, O. and C. Yucel. 2002. Different planting date and potassium fertility effects on cotton yield and fiber properties in the Çukurova region, Turkey. Field Crops Res., 78: 141-149.

Govt. of Pakistan (GOP), Economic Survey of Pakistan. 2020. Ministry of food, agriculture and livestock, Islamabad, pp. 17-22.

Hallikeri, S.S., H.L. Halemani, V.C. Patil, Y.B. Palled, B.C. Patil and I.S. Katageri. 2009. Influence of sowing time and moisture regimes on growth, seed cotton yield and fiber quality of Bt-cotton. Karnataka J. Agric. Sci., 22: 985991.

Hassan, M.U., M. Aamer, M.U. Chattha, M.A. Ullah, S. Sulaman M. Nawaz, W. Zhiqiang, M. Yanqin and H. Guoqin. 2017. The role of potassium in plants under drought stress: Mini review. J. Basic Appl. Sci., 13: 268-271. https:// doi.org/10.6000/1927-5129.2017.13.44

Hassan,M.U.,M.U.Chattha,A.Mahmood and S.T. Sahi. 2018. Performance of sorghum cultivars for biomass quality and biomethane yield grown in semi-arid area of Pakistan. Environ.
Sci. Poll. Res., 25(13): 12800-12807. https:// doi.org/10.1007/s11356-018-1575-4

Hassan, M.U., M.U. Chattha, M.B. Chattha, A. Mahmood and S.T. Sahi. 2019a. Chemical composition and methane yield of sorghum as influenced by planting methods and cultivars. J. Anim. Plant Sci., 29(1): 251-259.

Hassan, M.U., M.U. Chattha, L. Barbanti, M.B. Chattha, A. Mahmood, I. Khan and M. Nawaz. 2019b. Combined cultivar and harvest time to enhance biomass and methane yield in sorghum under warm dry conditions in Pakistan. Indus. Crops Prod., 132: 84-91. https://doi. org/10.1016/j.indcrop.2019.02.019

Hassan, M.U., M.U. Chattha, L. Barbanti, A. Mahmood,M.B.Chattha,I.Khan,S.Mirza,S.A. Aziz, M. Nawaz and M. Aamer. 2020. Cultivar and seeding time role in sorghum to optimize biomass and methane yield under warm dry climate. Indus. Crops Prod., 145: 111983. https://doi.org/10.1016/j.indcrop.2019.111983

Hassan, M.U., M.U. Chattha, I. Khan, M.B. Chattha, L. Barbanti, M. Aamer, M.M. Iqbal, M. Nawaz, A. Mahmood, A. Ali and M.T. Aslam M.T, 2020a. Heat stress in cultivated plants: Nature, impact, mechanisms, and mitigation strategies-a review. Plant Biosys. 155: 211-234. https://doi.org/10.1080/112635 04.2020 .1727987

Hassan, M.U., M. Aamer, M.U. Chattha, T. Haiying, B. Shahzad, L. Barbanti, M. Nawaz, A. Rasheed, A. Afzal, Y. Liu and H. Guoqin. 2020b. The critical role of zinc in plants facing the drought stress. Agriculture, 10(9): 396. https://doi.org/10.3390/agriculture10090396

Homer, D.C. and P.F. Pratt. 1961. Methods of analysis for soils, plants and waters. Davis: University of California, Davis.

Huang, J. and F. Ji. 2015. Effects of climate change on phenological trends and seed cotton yields in oasis of arid regions. Int. J. Biometeorol., 59: 877-888. https://doi.org/10.1007/s00484-0140904-7

Ilyas, M., I. Khan, M.U. Chattha, M.U. Hassan, M. Zain, W. Farhad, S. Ullah, A. Shah, S. Ahmed, B. Khan and M. Adeel. 2020. Evaluating the effect of zinc application methods on growth and yield of wheat cultivars. J. Innov. Sci., 6(2): 150-156. https://doi.org/10.17582/journal. jis/2020/6.2.150.156

Jordan, A.G., 2001. Management to increase 
strength. In: Cotton physiology today Newsletter. Natl. Cotton Counc. Am., 12: 1-8.

Karavina, C., R. Mandumbu, C. Parwada and T. Mungunyana. 2012. Variety and planting date effects on the incidence of bollworms and insect sucking pests of cotton (Gossypium hirsutum L.). Res. J. Agric. Sci., 3(3): 607-610.

Moser H.S., M. Closkey, J.C Silvertooth and P. Dugger, 2000. Performance of transgenic cotton varieties in Arizona. Proc. Beltw. Cotton Conf. San Antonio, USA, 1: 497- 499.

Muhsin, M., M. Nawaz, I. Khan, M.B. Chattha, S. Khan, M.T. Aslam, M.M. Iqbal, M.Z. Amin, M.A. Ayub, U. Anwar, M.U. Hassan and M.U. Chattha. 2021. Efficacy of seed size to improve field performance of wheat under late sowing conditions. Pak. J. Agric. Res., 34(1): 247-253. https://doi.org/10.17582/journal. pjar/2021/34.1.247.253

Nasim, W. 2010. Modeling the impact of climate change on nitrogen use efficiency in sunflower (Helianthus annuus L.) under different agroclimatic conditions of Punjab-Pakistan. PhD Thesis, University of Agriculture FaisalabadPakistan. Pp. 90-121.

Nuti, R.C., R.P.Viator, S.N. Casteel, K.L.Edmisten and R. Wells. 2006. Effect of planting date, mepiquat chloride, and glyphosate application to glyphosate-resistant cotton. Agron. J., 98: 1627-1633. https://doi.org/10.2134/ agronj2005.0360

O’Berry, N.B., J.C. Faircloth, M.A. Jones, D.A. Herbert, A.O. Abaye, T.E. McKemie and C. Brownie. 2009. Differential responses of cotton cultivars when applying mepiquat pentaborate. Agron. J., 101: 25-31. https://doi.org/10.2134/ agronj2007.0333

Patil, D.V., D.B. Deosarkar and S.G. Patil. 2009. Study of Bt and non-Bt cotton hybrids for yields and quality characters under normal and delay-sown condition. J. Cotton Res. Dev., 23(2): 199-203.

Pedigo, L.P., 2004. Entomology and pest management. Prentice Hall, Englewood Cliffs.

Pettigrew, W.T., 2002. Improved yield potential with an early planting cotton production system. Agron. J., 94: 997-1003. https://doi. org/10.2134/agronj2002.0997

Rahman, H.R., S.A. Malik, M. Saleem and F. Hussain. 2007. Evaluation of seed physical traits in relation to heat tolerance in upland cotton. Pak. J. Bot., 39: 475-483.

Ramey, H.H. 1999. Classing of fiber. In C.W. Smith and J.T. Cothren (ed.) Cotton: origin, history, technology, and production. John Wiley and Sons, New York, pp. 709-727.

Reynolds, M. and R. Tuberosa. 2008. Translational research impacting on crop productivity in drought prone environments. Curr. Opin. Plant Biol., 11: 171-179. https://doi.org/10.1016/j. pbi.2008.02.005

Saddique, Q., H. Cai, W. Ishaque, H. Chen, H.W. Chau, M.U. Chattha M.U. Hassan, I. Khan, M and J. He. 2019. Optimizing the sowing date and irrigation strategy to improve maize yield by using CERES (Crop Estimation through Resource and Environment Synthesis)maize model. Agron., 9(2): 109. https://doi. org/10.3390/agronomy9020109

Shah, M.K.N., S.A. Malik, N. Murtaza, I. Ullah, H. Rahman, U. Younis. 2010. Early and rapid flowering coupled with shorter boll maturation period offers selection criteria for early crop maturity in upland cotton. Pak. J. Bot., 42: 3569-3576.

Steel, R.G.D., J.H. Torrie and D.A. Dickey. 1997. Principles and Procedures of Statistics: A biometrical Approach (3 ${ }^{\text {rd }}$ Edi). McGraw-Hill, New York.

Wanga, X., H. Yuru, D. Mingwei, X. Dongyong, L. Huaiyu, T. Xiaoli and L. Zhaohu. 2016. Effect of planting date and plant density on cotton traits as relating to mechanical harvesting in the yellow river valley region of China Xiangru. Field Crops Res., 198: 112-121. https://doi. org/10.1016/j.fcr.2016.09.010 